\title{
O conhecimento dos acadêmicos de medicina das instituições particulares de Belém sobre o acidente vascular encefálico
}

\author{
The knowledge of medical students of the Belém's private institutions about the stroke \\ El conocimiento de los estudiantes de medicina de las universidades particulares en \\ Belém sobre el accidente vascular cerebral
}

Ursula Tatiane de Farias Hounsell Almeida ${ }^{1 *}$, Thiago Celeira de Sousa ${ }^{1}$, Amanda Santos Duarte ${ }^{1}$, Manuella Anchieta Gouveia ${ }^{1}$, Vitória Maria Leal Coelho', Celina Cláudia Israel Sefer ${ }^{1}$.

\begin{abstract}
RESUMO
Objetivo: Avaliar o conhecimento dos acadêmicos de Medicina de instituições particulares sobre o acidente vascular encefálico. Métodos: Estudo transversal, analítico e quantitativo, no qual, foram aplicados questionários com perguntas diretas e respostas objetivas aos acadêmicos de Medicina das faculdades particulares do Município de Belém do Pará, cursando do $1^{\circ}$ ao $8^{\circ}$ período. Resultados: Foram analisados 301 questionários, sendo 160 do CESUPA e 141 da UNIFAMAZ. A diferença entre o número de alunos das instituições deve-se pelo fato que no Centro Universitário UNIFAMAZ o número de alunos por semestre é menor quando comparado ao CESUPA. Entretanto, tal diferença não interferiu na validade da análise estatística. Verificou-se um bom desempenho geral dos alunos perante a análise estatística, havendo diferença significativa apenas na questão que abordava a conduta a ser seguida nos casos de AVE. Conclusão: Os resultados demonstraram que os acadêmicos de Medicina das duas instituições apresentaram conhecimento significativo e equivalente sobre o acidente vascular encefálico.
\end{abstract}

Palavras-chave: Acidente Vascular Cerebral, Educação Médica, Conhecimento.

\begin{abstract}
Objective: to evaluate the knowledge of medical students of private institutions about stroke. Methods: Crosssectional, analytical and quantitative study, in which questionnaires with direct questions and objective answers were applied to medical students from the private schools of the city of Belém, Pará, from the first to the eighth period. Results: Three hundred and one questionnaires were analyzed, of which one hundred and sixty were from CESUPA and one hundred and forty-one from UNIFAMAZ. The difference between the number of students in the both educational institutions is due to the fact that in UNIFAMAZ the number of students per semester is lower the CESUPA. However, such difference did not affect the validity of the statistical analysis. There was a good general performance of the students on the statistical analysis, and there was a significant difference only in the question that dealt with the conduct to be followed in case of stroke. Conclusion: The results showed that medical students from both institutions had significant and equivalent knowledge about stroke.
\end{abstract}

Key words: Stroke, Medical Education, Knowledge.

\section{RESUMEN}

Objetivo: evaluar el conocimiento de los estudiantes de medicina en universidades particulares sobre el accidente cerebrovascular. Métodos: Estudio transversal, analítico y cuantitativo, en el que se aplicaron cuestionarios con preguntas directas y respuestas objetivas a estudiantes de medicina de las escuelas de medicina privadas en Belém do Pará, que asistieron del primer al octavo semestre. Resultados: Analizamos 301 cuestionarios, 160 de CESUPA y 141 de UNIFAMAZ. La diferencia entre el número de estudiantes en las escuelas se debe al hecho de que en el Centro Universitario UNIFAMAZ el número de estudiantes por semestre es menor en comparación con CESUPA. Sin embargo, dicha diferencia no afectó la validez del análisis estadístico. Hubo un buen desempeño general de los estudiantes antes del análisis estadístico, con una diferencia significativa solo en el tema que abordaba la conducta a seguir en los casos de accidente cerebrovascular. Conclusión: Los resultados mostraron que los estudiantes de medicina de ambas instituciones tenían un conocimiento significativo y equivalente sobre el accidente cerebrovascular.

Palabras clave: Accidente Cerebrovascular, Educación Médica, Conocimiento.

${ }^{1}$ Centro Universitário Metropolitano da Amazônia (UNIFAMAZ), Belém-Para. *E-mail: ursula.tati@ymail.com 


\section{INTRODUÇÃO}

O Acidente Vascular Encefálico (AVE) constitui causa importante de morte e de incapacidade no mundo. No Brasil, representa a terceira maior causa de internação e, anualmente cerca de 30\% dos sobreviventes ficam dependentes para as atividades de vida diária (MEIRA F, 2018; VAHDATI SS, 2017; KHUBAIB MU, 2016).

O AVE é dividido em duas categorias principais, isquêmico (AVEI) e hemorrágico (AVEH). O primeiro é o mais frequente, $85 \%$ dos casos, em geral por uma oclusão vascular seguida por hipoperfusão da área lesionada. Já o segundo é menos frequente, $15 \%$ dos casos, e decorre de extravasamento de sangue para o parênquima cerebral ou espaço subaracnóide (SOHAIL CS, 2018).

Os fatores de riscos associados ao AVE são: idade, raça, hereditariedade, tabagismo, hipertensão, diabetes mellitus, dislipidemia, uso de álcool e drogas ilícitas, obesidade e cardiopatias. A consciência de tais fatores e de seu controle é primordial para minimizar a incidência dessa patologia, e para isso a iniciativa educacional pelos profissionais da saúde para a população se faz importante (SOHAIL CS, 2018; KHUBAIB MU e LIMA-RAMOS MJM, 2016).

As manifestações clínicas, como parestesia, alteração da fala e/ou visão, acarretam prejuízo das funções sensitivas, motoras, de equilíbrio e de marcha, além do déficit cognitivo e de linguagem. $O$ déficit motor em decorrência do AVE pode levar a hemiplegia, caracterizada pela perda da força muscular progressiva de um hemicorpo e atrofia dos mesmos por desuso, fato este que propicia outros agravos a saúde do enfermo. Estas sequelas comprometem a qualidade de vida do paciente tanto funcional, emocional, como 0 autocuidado (KATO S, 2017; KHUBAIB MU e GOMES ABAGR, 2016).

Para o diagnóstico inicial do AVE, de acordo com o Ministério da Saúde (2013) é recomendado o uso do mnemônico SAMU com quatro ações básicas, dentre elas: pedir para o paciente sorrir; abrir os braços e cantar uma música. Se estes movimentos estiverem alterados deve-se ligar para o Serviço de Atendimento Móvel de Urgência (SAMU) imediatamente. Já a conduta na emergência para confirmação do diagnóstico envolve exames de imagens e laboratoriais (SOHAIL CS, 2018; VAHDATI SS, GOMES ABAGR e LIMARAMOS MJM, 2016).

Quanto ao tratamento na emergência, este irá depender do tipo da lesão, se isquêmica ou hemorrágica. Vale ressaltar que para o AVE isquêmico é recomendado o uso de ativador do plasminogênio tecidual recombinante (rt-PA), se dentro do tempo ideal para sua aplicabilidade de $4,5 \mathrm{~h}$ do início dos sintomas (SOHAIL CS, 2018; VAHDATI SS e LOCATELLI MC, 2017; SUDHA MJ, 2016).

Considerando a repercussão da doença não apenas para o enfermo, mas também para a família e a para a saúde pública, é importante que o paciente seja atendido e encaminhado para um hospital preferivelmente dentro do período de 4,5 horas, visando reduzir os danos e as sequelas que podem causar no sistema nervoso central. No entanto, a inabilidade do reconhecimento dos sintomas é uma das maiores causas da demora da busca do serviço de emergência (SUDHA MJ e THAPA L, 2016).

Alguns países como os Estados Unidos da América vêm implementando programas de conscientização sobre os sinais e sintomas de AVE tanto para a população quanto para os acadêmicos dos cursos da saúde. Associado a isso, países desenvolvidos vêm trabalhando também na prevenção da atenção básica primária visando reduzir/controlar os fatores de risco para AVE (SUDHA MJ e THAPA L, 2016).

Diante do exposto, essa pesquisa visa determinar o conhecimento dos acadêmicos de Medicina em relação ao AVE, a fim de propagar a conduta inicial adequada que possibilite reduzir os danos e sequelas desta doença.

\section{MÉTODOS}

Estudo transversal, analítico e quantitativo, no qual, foram aplicados questionários com perguntas diretas e respostas objetivas aos acadêmicos de Medicina do Centro Universitário do Pará (CESUPA) e Centro Universitário Metropolitano da Amazônia (UNIFAMAZ) do Município de Belém do Pará no período de abril a maio de 2019. 
O presente estudo foi aprovado pelo Comitê de Ética em Pesquisa (CEP) do Centro Universitário Metropolitano da Amazônia (UNIFAMAZ), segundo os preceitos da declaração de Helsinque (1975) e do código de Nuremberg, respeitadas as normas regulamentadoras de pesquisa envolvendo seres humanos (Res.CNS466/12) do Conselho Nacional de Saúde pelo número: CAAE: 02823018.5.0000.5701.

Todos os acadêmicos incluídos na pesquisa foram avaliados segundo os preceitos éticos após a assinatura do Termo de Consentimento Livre e Esclarecido (TCLE), garantindo anonimato e segurança aos participantes. Compuseram a amostra 301 acadêmicos de Medicina, que estavam regularmente matriculados do primeiro ao oitavo semestre do curso no período da pesquisa. O recrutamento dos alunos foi feito por meio de convite nas salas de aula, após explanação prévia sobre os objetivos, métodos, riscos e benefícios.

Foram excluídos aqueles que desistiram do curso ao longo da pesquisa e os que responderam os questionários de forma incompleta. A seleção da amostra foi feita de forma voluntária e aleatória pelos pesquisadores aos estudantes. Do universo de 1030 alunos, destes, 301 foram incluídos com sucesso.

Para a coleta de dados foram aplicados questionários que avaliaram o conhecimento dos acadêmicos sobre o Acidente Vascular Encefálico e também permitiu avaliar o perfil da amostra quanto ao semestre e a instituição a qual fazia parte.

O questionário era composto por seis perguntas objetivas acerca dos tipos de AVE, os fatores de risco, os sinais e sintomas, as incapacidades, os fatores preventivos e a solicitação de ajuda. Dentre as opções de resposta foram apresentados apenas uma assertiva correta ao item pesquisado.

O questionário fora elaborado pelos autores desta pesquisa, tomando como base as escalas de National Institute of Health Stroke Scale (NIH), escala de Rankin e Manual do Ministério da Saúde sobre rotinas para atenção ao AVC (MINISTÉRIO DA SAÚDE BR, 2013; NIH EUA, 1999; RANK EUA,1997).

A partir dos dados coletados, os questionários foram separados em grupos: inicialmente do $1^{\circ}$ ao $4^{\circ}$ semestre e posteriormente do $5^{\circ}$ ao $8^{\circ}$ semestre. Após a ponderação sobre os dados, foi elaborado uma cartilha explicativa sobre o manejo do AVE para distribuição entre os acadêmicos dos semestres avaliados das faculdades participantes da referida pesquisa.

O tamanho amostral foi determinado pelo método probabilístico ao nível de significância de $5 \%$ de erro amostral. As informações da caracterização amostral foram apuradas em banco de dados elaborado no software Microsoft ${ }^{\circledR}$ Office Excel® 2016. Na aplicação da estatística descritiva, foram construídos tabelas e gráficos para apresentação dos resultados e calculadas as medidas de posição como média aritmética e desvio padrão.

A estatística analítica foi utilizada para avaliar os resultados das variáveis categóricas da amostra através do Teste Qui-Quadrado Aderência para tabelas univariadas e Independência para as comparações bivariadas. As estatísticas descritiva e analítica, foram realizadas no software BioEstat $\AA^{\circledR}$ 5.3. Para a tomada de decisão, adotou-se o nível de significância $\alpha=0,05$ ou $5 \%$, sinalizando com asterisco $\left({ }^{*}\right)$ os valores significantes (PRODANOV CC, 2013).

\section{RESULTADOS}

Foram analisados 301 questionários, sendo 160 do CESUPA e 141 da UNIFAMAZ. A diferença entre o número de alunos das instituições deve-se pelo fato que no Centro Universitário UNIFAMAZ o número de alunos por semestre é menor quando comparado ao CESUPA. Entretanto, tal diferença não interferiu na validade da análise estatística.

$\mathrm{Na}$ comparação dos percentuais de acertos, no geral, em cada questão, é possível observar uma equivalência entre as instituições de ensino superior (IES), já que, cinco das seis questões abordadas não apresentaram diferença estatisticamente significante. A última questão, sobre o que fazer em caso de ocorrência de um AVE, foi a única que apresentou diferença significante em seus percentuais $(p=0,0284)$, sendo a UNIFAMAZ a instituição com maior percentual de acertos entre os discentes abordados. 


\section{Desempenho no item identificação dos tipos de AVE}

Os discentes das IES alcançaram percentual de acertos semelhante, independente dos semestres cursados. Mesmo com o baixo percentual de acertos referente ao primeiro período do CESUPA em relação a UNIFAMAZ, tal diferença não se mostrou estatisticamente significante $(p=0.7800)$ quando comparados todos os períodos (Gráfico 1).

Gráfico 1 - Acertos na identificação dos tipos de Acidente Vascular Encefálico de acordo com a IES e o semestre cursado, $p=0.7800$. Belém, 2019.

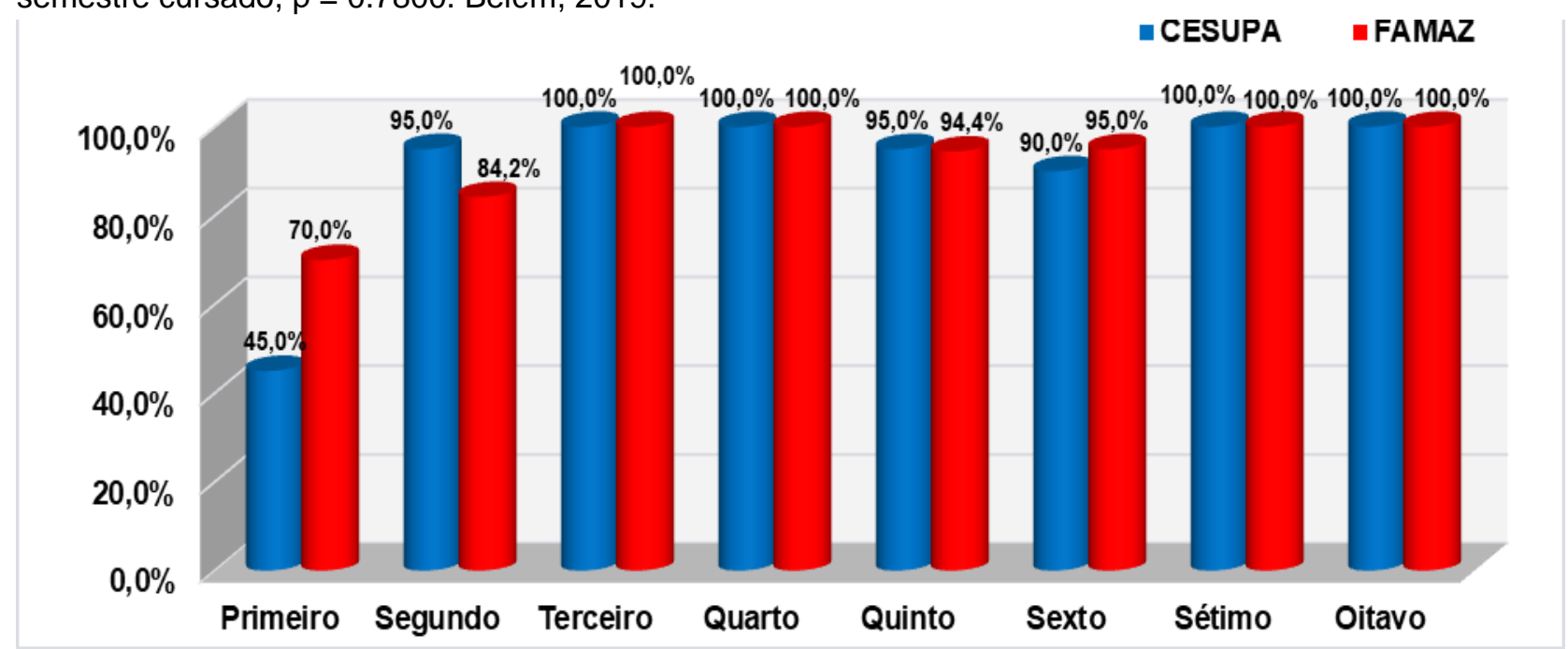

Fonte: Almeida UTFH, et al., 2019.

\section{Desempenho no item fatores de risco}

Não se mostrou como dificuldade para os discentes de ambas IES. Alunos do segundo período da UNIFAMAZ foram os que apresentaram maior diferença percentual de acertos entre os períodos avaliados $(84,2 \%)$, mas este valor não teve relevância estatística quando comparado aos demais períodos testados ( $p$ $=0.2038)($ Gráfico 2).

Gráfico 2 - Acertos na identificação dos fatores de risco para Acidente Vascular Encefálico de acordo com a IES e o semestre cursado, $p=0.2038$. Belém, 2019.

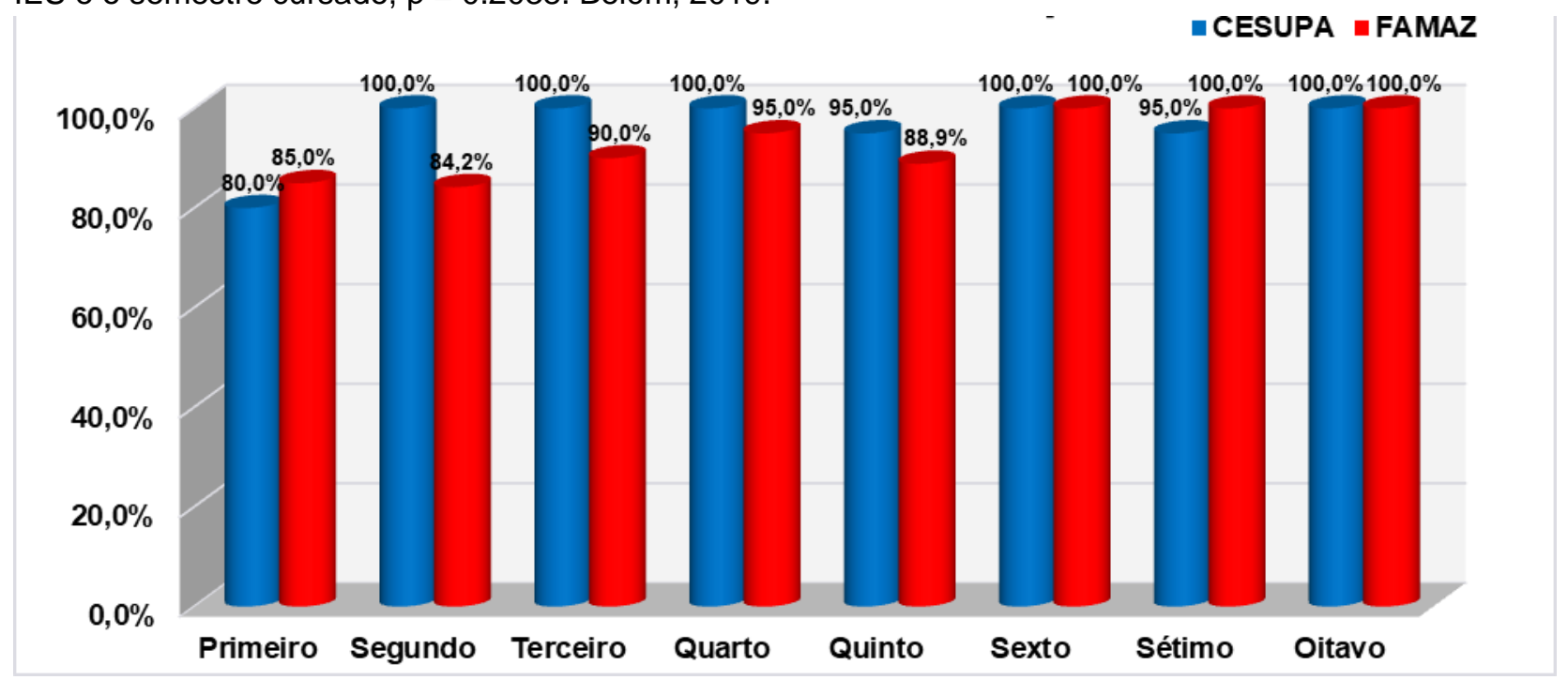

Fonte: Almeida UTFH, et al., 2019. 


\section{Desempenho no item sinais e sintomas}

Os discentes apresentaram conhecimento no caso de vivência desta situação. O menor percentual de acertos alcançado foi de $65,0 \%$, pelos discentes do primeiro semestre do CESUPA, não influenciando no resultado final que não apresentou diferença estatisticamente significante entre os semestres $(p=0.7627)$ (Gráfico 3).

Gráfico 3 - Acertos na identificação dos sinais e sintomas sugestivos de Acidente Vascular Encefálico de acordo com a IES e o semestre cursado, $\mathrm{p}=0.7627$. Belém, 2019.

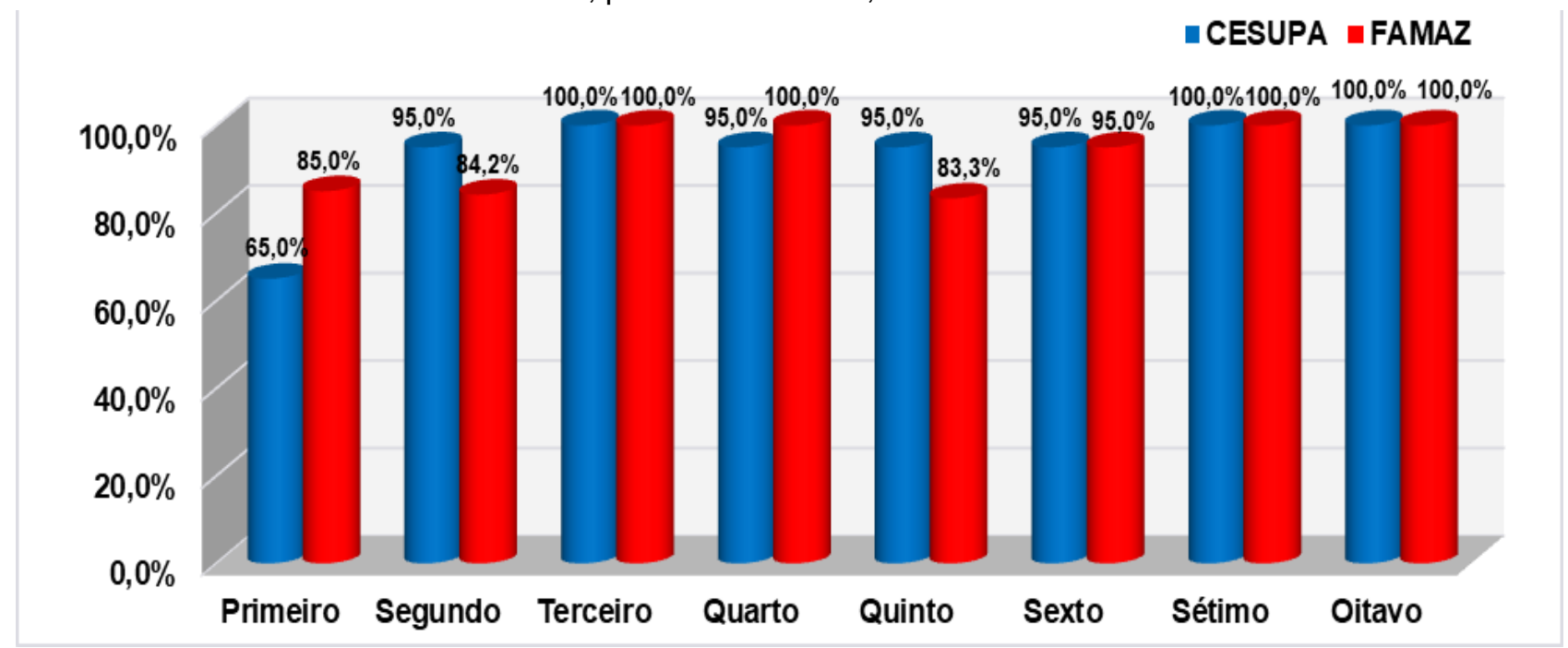

Fonte: Almeida UTFH, et al., 2019.

\section{Desempenho no item incapacidades}

Os alunos apresentaram percentis equivalentes nesse quesito. O primeiro semestre do CESUPA alcançou um percentual inferior de acertos $(75,0 \%)$, quando comparado ao semestre equivalente da UNIFAMAZ (90.0\%). O mesmo desempenho inferior fora visto no sétimo semestre da UNIFAMAZ em relação ao CESUPA, o que contribuiu para que a comparação entre os semestres, na análise geral, não se mostrasse estatisticamente significante $(p=0.8785)$ (Gráfico 4).

Gráfico 4 - Acertos na identificação das incapacidades decorrentes do Acidente Vascular Encefálico de acordo com a IES e o semestre cursado, $p=0.8785$. Belém, 2019

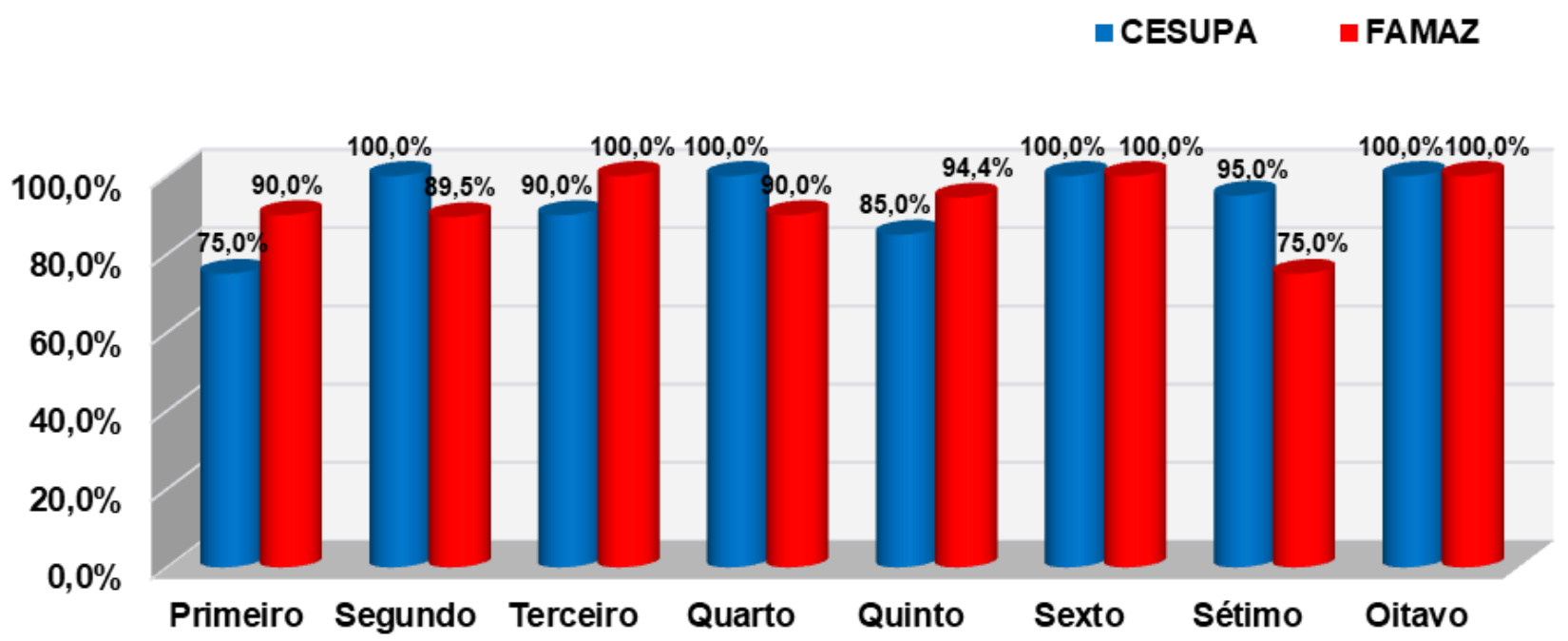

Fonte: Almeida UTFH, et al., 2019. 


\section{Desempenho no item critérios de prevenção}

Este quesito alcançou um bom percentual de acertos entre os discentes, 95,6\% e 94,3\%. Nesta abordagem, o segundo período da UNIFAMAZ, foi o que mostrou menor conhecimento com um percentual baixo de acertos (73,7\%). Também não foi encontrada diferença estatisticamente significante entre os períodos comparados $(p=0.8016)$ (Gráfico 5).

Gráfico 5 - Acertos na identificação dos critérios de prevenção do Acidente Vascular Encefálico de acordo com a IES e o semestre cursado, $p=0.8016$. Belém, 2019.

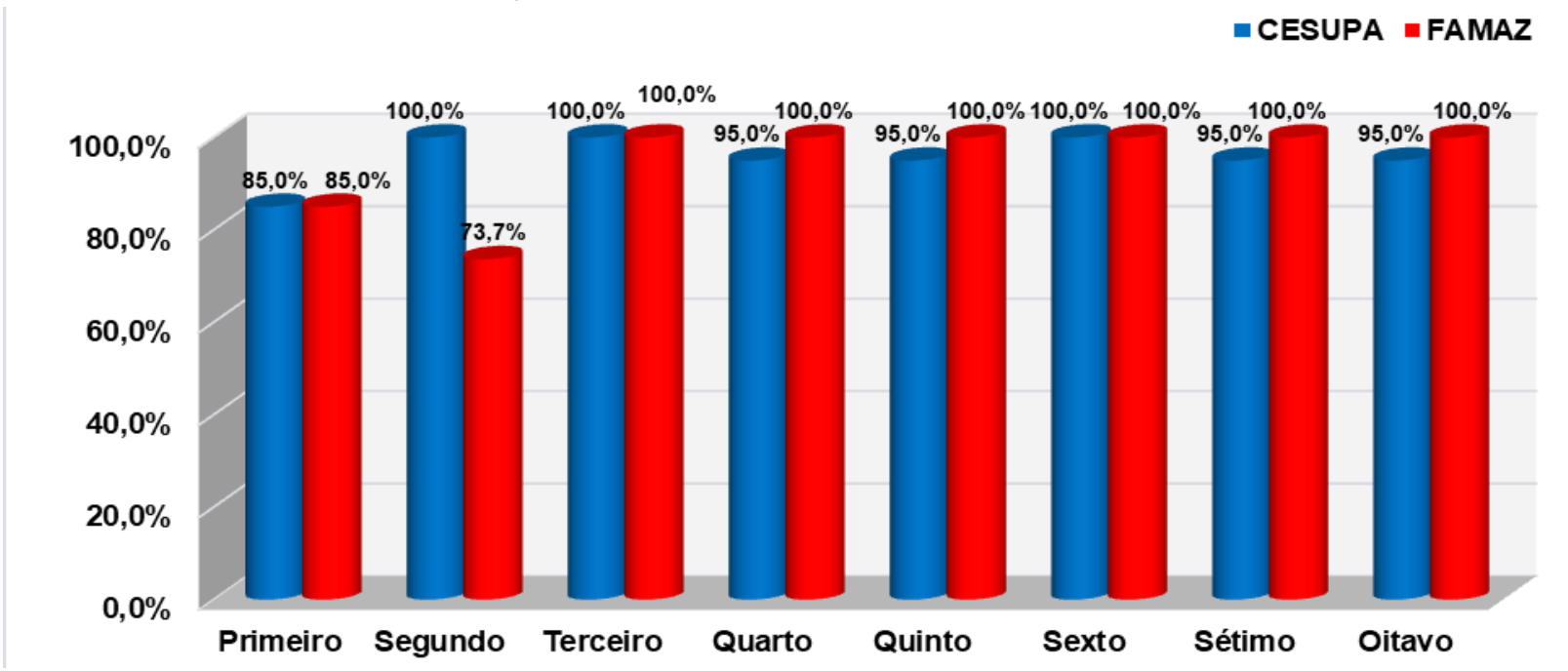

Fonte: Almeida UTFH, et al., 2019.

\section{Desempenho no item conduta a seguir}

Neste quesito os alunos da UNIFAMAZ apresentaram maior percentual de acertos $(92,2 \%)$ em relação ao CESUPA (83,1\%). Apenas o quarto período da UNIFAMAZ foi inferior $(80,0 \%)$ ao CESUPA (90,0\%). Já em relação aos demais períodos do CESUPA, todos apresentaram um menor percentual de acertos quando comparados aos da UNIFAMAZ, com exceção do oitavo período que apresentou $100,0 \%$ em ambas as IES. Esse fato fez com que a diferença entre os semestres se mostrasse estatisticamente significante $(p=$ 0.0180) (Tabela 1).

Tabela 1 - Acertos na identificação de que conduta seguir em caso de Acidente Vascular Encefálico de acordo com a IES e o semestre cursado, ${ }^{*} p=0.0180$. Belém, 2019.

\section{SABE O QUE FAZER EM CASO DE AVE}

\begin{tabular}{ccccc}
\hline Período & \multicolumn{2}{c}{ CESUPA $(\mathbf{n}=\mathbf{1 6 0})$} & \multicolumn{2}{c}{ UNIFAMAZ (n = 141) } \\
\hline \hline Primeiro & 15 & $75.0 \%$ & 17 & $85.0 \%$ \\
Segundo & 16 & $80.0 \%$ & 18 & $94.7 \%$ \\
Terceiro & 17 & $85.0 \%$ & 20 & $100.0 \%$ \\
Quarto & 18 & $90.0 \%$ & 16 & $80.0 \%$ \\
Quinto & 15 & $75.0 \%$ & 17 & $94.4 \%$ \\
Sexto & 14 & $70.0 \%$ & 19 & $95.0 \%$ \\
Sétimo & 18 & $90.0 \%$ & 11 & $91.7 \%$ \\
Oitavo & 20 & $100.0 \%$ & 12 & $100.0 \%$ \\
\hline TOTAL & $\mathbf{1 3 3}$ & $\mathbf{8 3 . 1 \%}$ & $\mathbf{1 3 0}$ & $\mathbf{9 2 . 2} \%$ \\
\hline \hline
\end{tabular}

Fonte: Almeida UTFH, et al., 2019. 


\section{DISCUSSÃO}

Os semestres foram escolhidos de acordo com o Plano Pedagógico do Curso (PPC) de Medicina da UNIFAMAZ e CESUPA, dessa forma, explica-se a evolução do conhecimento e melhor desempenho apresentado pelos discentes sobre o tema no decorrer do curso. Uma vez que, ao final do 8 semestre os alunos já cursaram o módulo de neurologia e por isso obtiveram melhor desempenho em relação aos demais semestres avaliados.

No item a identificação dos tipos de Acidente Vascular Encefálico os primeiros semestres de ambas IES apresentaram menor percentil de acertos quando comparado aos demais. O que já era esperado, pois $O$ módulo de Neurologia é abordado a partir do 4ํㅗㅇ semestre na UNIFAMAZ e $2^{\circ}$ semestre no CESUPA. Os demais períodos souberam responder corretamente à questão alcançando percentis de 90,6\% CESUPA e 92,2\% UNIFAMAZ, demonstrando o reconhecimento da importância do tema para a prática médica. Reconhecer os tipos de AVE proporciona melhor conduta e direcionamento para o tratamento mais adequado para cada forma de apresentação (LOCATELI MC, 2017).

Quanto ao reconhecimento dos fatores de risco, observa-se menor número de acerto entre os acadêmicos dos primeiros semestres das duas instituições e segundo semestre da UNIFAMAZ, que se justifica pelo PPC do curso visto que, o módulo de Neurologia ainda não fora abordado. Este conhecimento se faz necessário para que os alunos possam correlacionar os fatores de risco à patologia. São referidos como fatores de risco na literatura a idade avançada, as dislipidemias, diabetes mellitus, hipertensão arterial sistêmica, tabagismo, sedentarismo e as doenças cardiovasculares (LOCATELI MC, 2017; PORTELA MB, 2017; CALDATO MCF,2016).

No que se refere à identificação dos sinais e sintomas, grande parte dos acadêmicos de ambas instituições se mostraram conhecedores das possíveis manifestações clínicas do AVE, alcançando percentual de 93,1\% CESUPA e 92,9\% UNIFAMAZ. A identificação da sintomatologia auxilia e promove a busca por atendimento médico mais rápido e com isso a possibilidade de redução de sequelas por meio do tratamento adequado, se dentro do tempo para abordagem. O reconhecimento precoce dos sintomas é primordial para solicitação de ajuda junto à emergência, possibilitando um desfecho menos debilitante na evolução da doença (GOMES ABAGR, 2016).

Outro dado importante a se ressaltar quanto a identificação dos sinais e sintomas é que em ambas as faculdades, o sétimo e oitavo períodos apresentaram 100\% de acerto nesse quesito. Este resultado demonstra que o aprendizado em espiral proposto pela metodologia de ensino das faculdades é factível, pois os alunos obtiveram o conhecimento em semestres anteriores e reveem o assunto no sétimo período na UNIFAMAZ e no oitavo período no CESUPA, além do fato de já estarem vivenciando a prática hospitalar o que auxilia na sedimentação do conhecimento por associar teoria e prática (CALDATO MCF, 2016).

Quanto à identificação das incapacidades decorrentes do Acidente Vascular Encefálico, o percentil de acertos entre os acadêmicos das duas instituições avaliadas mostrou-se equivalentes. Sendo o AVE uma das principais causas de incapacidade física e neurológica no mundo, é de grande importância o reconhecimento a respeito das possíveis sequelas que esta patologia pode acarretar a vida do doente (VAHDATI SS, 2017).

Ao analisar o percentual de acertos entre os semestres observa-se que o primeiro semestre do CESUPA e o sétimo semestre da UNIFAMAZ obtiveram percentil de $75 \%$, quando comparado aos demais semestres. Valores inferiores de acertos eram esperados para os semestres iniciais de ambas as instituições. Entretanto, o mesmo não era esperado para o sétimo semestre UNIFAMAZ, uma vez que os acadêmicos já obtiveram esse conhecimento no quarto e sétimo períodos do curso nas sessões tutoriais e nas habilidades clínicas (PORTELLA MB, 2017; CALDATO MCF, 2016).

Segundo o PPC UNIFAMAZ, no quarto período do curso de Medicina, os acadêmicos iniciam os conhecimentos acerca da Neurologia no eixo Atenção e Educação em Saúde com os seguintes conteúdos: Somestesia, sistema neuroendócrino e neuroimunológico. Tais conhecimentos são aprimorados no eixo habilidades clínicas 4 quando os alunos podem aplicar na prática o aprendizado adquirido nas sessões 
tutoriais. Os conteúdos abordados nas habilidades clínicas são: Anamnese; exame físico; e pesquisa de sinais neurológicos especiais (PORTELLA MB, 2017; CALDATO MCF, 2016).

No sétimo período os acadêmicos retomam a abordagem neurológica no eixo Atenção e Educação em Saúde, abrangendo os seguintes conteúdos: Síndromes piramidais e extrapiramidais; doenças cerebrovasculares; doenças desmielinizantes; mielopatias; polineuropatias; epilepsia; distúrbios do movimento; miopatias; métodos diagnósticos usados na Neurologia. Ainda no mesmo período de curso, no eixo Habilidade Clínicas 7, o módulo de Neurologia abrange o atendimento ambulatorial com ênfase aos distúrbios neurológicos abordando o exame neurológico completo; doenças cérebro-vasculares; cefaleia; doenças desmielinizantes; síndromes demenciais; polineuropatias; epilepsia; distúrbios do movimento; métodos diagnósticos; interpretação dos exames; diagnóstico diferencial; tratamento e reabilitação (PORTELLA MB, 2017; CALDATO MCF, 2016).

Portanto, os resultados obtidos no sétimo período da UNIFAMAZ não fora o esperado, uma vez que, o aprendizado por metodologias ativas na forma de espiral proporciona a sedimentação do conhecimento conforme o avançar dos módulos, o que contrapõe alguns estudos (GARCÍA RR e MEIRA F, 2018).

No que tange a prevenção do AVE, ao analisar o desempenho dos alunos percebeu-se que tanto os primeiros semestres das faculdades como o segundo semestre UNIFAMAZ foram os períodos com os menores acertos.

Entretanto, a análise geral entre as escolas mostra o conhecimento equivalente com percentis de 95,6\% CESUPA e 94,3\% UNIFAMAZ. Esses dados corroboram com outros estudos, pois métodos preventivos como controle do peso corporal, alimentação saudável, a prática de atividade física e controle pressórico estão entre a prevenção de outras patologias, o que auxilia para o fortalecimento do aprendizado (ARAÚJO JP, 2018).

Quanto à conduta a seguir em caso de suspeita de AVE. Esperava-se que todos os alunos soubessem que deveria entrar em contato com o SAMU-192 para obtenção de auxílio apropriado. Entretanto, apenas o terceiro e oitavo período da UNIFAMAZ e o oitavo período do CESUPA obtiveram $100 \%$ de acerto na resposta. Este resultado é preocupante, pois os estudantes de medicina são os que mais deveriam deter deste conhecimento, uma vez que eles podem perpetuar esta informação para a população leiga. Além disso, acarretará um atraso significante na conduta do paciente, podendo aumentar a chance de sequelas e diminuir a sobrevida (LIMA-RAMOS MJM, 2016; MINISTÉRIO DA SAÚDE BR, 2013).

\section{CONCLUSÃO}

Os resultados demonstraram que os acadêmicos de Medicina das duas instituições apresentaram conhecimento significativo e equivalentes sobre o acidente vascular encefálico. $\mathrm{Na}$ comparação dos percentuais de acertos, no geral, em cada questão, é possível observar uma equivalência entre as instituições de ensino superior, já que cinco, das seis questões abordadas, não apresentaram diferença estatisticamente significante. Diante dos dados obtidos, verifica-se a necessidade da divulgação de informações sobre este assunto, visto que este estudo foi limitado e regional, necessitando de outros para corroborar ou refutar os dados.

\section{REFERÊNCIAS}

1. ARAÚJO JP, et al. Tendência da Mortalidade por Acidente Vascular Cerebral no Município de Maringá, Paraná entre os anos de 2005 a 2015. International Journal of Cardiovascular Sciences, 2018; 31(1): 56-62.

2. CALDATO MCF. Projeto Pedagógico do Curso de Medicina - CESUPA. Belém: CESUPA, 2016; (Série Material Instrucional do CESUPA).

3. GARCIA RR, et al. Response to Symptoms and Prehospital Delay in Stroke Patients. Is It Time to Reconsider Stroke Awareness Campaigns? J Stroke Cerebrovasc Dis, 2018; 27(3): 625-632.

4. GOMES ABAGR, et al. Popular stroke knowledge in Brazil: A multicenter survey during "WorldStroke Day". NeurologicalSci, 2016; 16(6): 63-67. 
5. KATO S, et al. Effects of a school-based stroke education program on stroke-related knowledge and behaviour modification-school class-based intervention study for elementary school students and parental guardians in a Japanese rural area. BMJ Open, 2017; 7: e17632.

6. KHUBAIB MU, et al. Knowledge Regarding Basic Facts of Stroke Among Final Year MBBS Students and House Officers: A Cross-Sectional Survey of 708 Respondents from Pakistan. Cureus, 2016; 8(3): e539.

7. LIMA-RAMOS MJM, et al. Fatores associados ao conhecimento dos adultos jovens sobre histórico familiar de Acidente Vascular Cerebral. Rev. Latino-Am. Enfermagem, 2016; 24: e2814.

8. LOCATELLI MC, et al. Perfil epidemiológico dos pacientes com acidente vascular cerebral isquêmico atendidos em um hospital. Rev Soc Bras Clin Med, 2017; 15(3): 150-4.

9. PORTELLA MB. Projeto Pedagógico do Curso de Medicina - UNIFAMAZ. Belém: UNIFAMAZ, 2017; (Série Material Instrucional da UNIFAMAZ).

10. MEIRA F, et al. Knowledge about Stroke in Belo Horizonte, Brazil: A Community-Based Study Using na Innovative Video Approach. Cerebrovasc Dis Extra, 2018; 8:60-69.

11. MINISTÉRIO DA SAÚDE (BR). Secretaria de Atenção à Saúde (BR). Departamento de Atenção Especializada. Manual de rotinas para atenção ao AVC. Brasília: Editora do Ministério da Saúde, 2013.

12. NIH STROKE SCALE INTERNATIONAL. EUA: The Association. 1999 [Citado 2019 May 11]. NIH Stroke Scale International; [about 2 screens].

13. PRODANOV CC, FREITAS EC. Metodologia do Trabalho Científico: métodos e técnicas da pesquisa e do trabalho acadêmico. 2 ed. Rio Grande do Sul: Universidade Feevale, 2013; 24p.

14. SOHAIL CS, et al. Basic Life Support: Knowledge and Attitude of Medical Students. Ann Community Med Pract, 2018; 4(2): 1034.

15. SUDHA MJ, et al. Assessment of prescription writing skills among undergraduate medical students. International Journal of Basic and Clinical Pharmacology, 2016; 5(4): 1586-93.

16. THAPA L, et al. Knowledge, attitude, and practice of stroke among high school students in Nepal. J Neurosci Rural Pract, 2016; 7(4): 504-509.

17. THE INTERNET STROKE CENTER. Washington. 1997-2019 [Citado 2019 May 11]. The Internet Stroke Center; [1 screen].

18. VAHDATI SS, et al. Knowledge and Skills of Medical Students about Stroke Patients: A Review. Advances in Bioscience and Clinical Medicine, 2017; 5(3): 10-28. 